OPEN ACCESS

Edited by:

Ulrike Kappler,

The University of Queensland,

Australia

Reviewed by:

Mohammad Roghanian,

Umeå University, Sweden

Cheng-Cai Zhang,

Chinese Academy of Sciences (CAS),

China

${ }^{*}$ Correspondence:

Rupak K. Bhadra

rupakbhadra@iicb.res.in

Bhabatosh Das

bhabatosh@thsti.res.in

tPresent address:

Shreya Dasgupta,

Clinical Medicine Division,

ICMR-National Institute of Cholera and Enteric Diseases, Kolkata, India

Specialty section:

This article was submitted to Microbial Physiology and Metabolism,

a section of the journa

Frontiers in Microbiology

Received: 22 May 2020 Accepted: 08 September 2020 Published: 29 September 2020

Citation:

Rakshit D, Dasgupta S, Das B and Bhadra RK (2020) Functional Insights Into the Role of gppA in (p)ppGpp

Metabolism of Vibrio cholerae.

Front. Microbiol. 11:564644. doi: 10.3389/fmicb.2020.564644

\section{Functional Insights Into the Role of gppA in (p)ppGpp Metabolism of Vibrio cholerae}

\author{
Dipayan Rakshit ${ }^{1}$, Shreya Dasgupta ${ }^{1 \dagger}$, Bhabatosh Das ${ }^{2 *}$ and Rupak K. Bhadra ${ }^{1 *}$ \\ ${ }^{1}$ Infectious Diseases and Immunology Division, CSIR-Indian Institute of Chemical Biology, Kolkata, India, ${ }^{2}$ Molecular \\ Genetics Laboratory, Infection and Immunology Division, Translational Health Science and Technology Institute, NCR Biotech \\ Science Cluster, Haryana, India
}

The stringent response, an adaptive response to nutrient limitation and exposure to xenobiotics in bacteria, is mediated by two intracellular signaling molecules, pppGpp and ppGpp, together called (p)ppGpp. The cellular level of (p)ppGpp in bacterial cells is controlled by the Rel/Spo family of proteins. In the cholera pathogen, Vibrio cholerae, (p)ppGpp metabolism is regulated by the products of at least three genes relA, spoT, and $r e / V$. In this study, we identify and characterize the function of the guanosine$5^{\prime}$-triphosphate $3^{\prime}$-diphosphate pyrophosphatase A (GppA) encoding gene gppA of $V$. cholerae. Genomic analysis indicates that the gppA locus is conserved in vibrios and organized as a bicistronic operon along with the rhlB gene. We engineered the genome of $V$. cholerae to develop different mutants devoid of GppA and/or other phosphate metabolic enzymes. Our findings indicate that in $V$. cholerae, GppA plays an important role in the conversion of pppGpp to ppGpp during amino acid deprivation but not during glucose starvation. Quantitative analyses of the gppA transcript level reveal its differential expression pattern at different growth phases and starvation conditions. It has been observed that the GppA deficiency during amino acid starvation condition could be complemented by overexpressing the exopolyphosphatase coding gene ppx of $V$. cholerae. By deletion analysis, we further demonstrate that the amino and carboxy terminal sequences flanking the Ppx-GppA motif of the GppA protein of $V$. cholerae are also important for its enzymatic function.

Keywords: Vibrio cholerae, stringent response, (p)ppGpp, GppA, Ppx, amino acid starvation, glucose starvation

\section{INTRODUCTION}

The enteric pathogen Vibrio cholerae faces various physicochemical stresses while living within or outside of the human intestine. Among multiple environmental stresses, nutritional stress is important for survival since it determines the growth and multiplication of the pathogen under such conditions. Like other bacterial pathogens, $V$. cholerae has evolved with complex gene regulatory networks to cope with various environmental stresses, among which the stringent response (SR) is important. The SR is typically characterized by strong repression of transcription of stable RNAs like transfer RNAs (tRNAs) and ribosomal RNAs (rRNAs), etc. (Cashel et al., 1996; Potrykus and Cashel, 2008; Gaca et al., 2015), upregulation of transcription of genes coding for the enzymes involved in amino acid biosynthesis (Stephens et al., 1975; Choy, 2000), and 
inhibition of replication (Wang et al., 2007). Regulations of all the above stated cellular processes under nutritional stress conditions are crucial for the survival of bacteria. The SR is essentially managed by two intracellular small signaling molecules, guanosine 3'-diphosphate 5'-triphosphate (pppGpp) and guanosine 3',5'-bis(diphosphate) (ppGpp), together called (p)ppGpp (Cashel et al., 1996; Potrykus and Cashel, 2008). While in Escherichia coli, intracellular (p)ppGpp metabolism is controlled by two multidomain containing proteins RelA and SpoT, in $V$. cholerae, apart from these two enzymes, a small alarmone synthetase, called RelV, is also involved in (p)ppGpp metabolism as shown in Figure 1 (Haralalka et al., 2003; Das and Bhadra, 2008; Das et al., 2009; Dasgupta et al., 2014). RelA, the product of the relA gene, is a ribosome-associated protein and responsible for (p)ppGpp synthesis under amino acid starvation (Cashel et al., 1996). RelA is able to synthesize pppGpp and ppGpp by using guanosine triphosphate (GTP) and guanosine diphosphate (GDP) as substrates, respectively (Mechold et al., 2002; Sajish et al., 2009; Kudrin et al., 2018). On the other hand, SpoT, the product of the spoT gene, has a strong (p)ppGpp hydrolase activity and weak (p)ppGpp synthetase activity under different stress conditions (Xiao et al., 1991; Seyfzadeh et al., 1993;
Das et al., 2009). Interestingly, in $V$. cholerae, the RelV enzyme, which is unique among Gram-negative bacteria, also contributes in (p)ppGpp synthesis under glucose and fatty acid starvations (Das et al., 2009; Dasgupta et al., 2014). Furthermore, unlike RelA and SpoT, RelV is a small single-domain-containing protein (Das et al., 2009; Dasgupta et al., 2014). In E. coli, apart from RelA and SpoT, another well-characterized enzyme, called guanosine pentaphosphate $5^{\prime}$-phosphohydrolase A (GppA), is associated with the (p)ppGpp metabolic cycle (Somerville and Ahmed, 1979; Hara and Sy, 1983; Keasling et al., 1993; Mechold et al., 2013). The main function of GppA is to convert pppGpp to ppGpp by removing the terminal $\gamma$-phosphate from 5 '-position of pppGpp. Initial mutational analysis by Somerville and Ahmed (1979) indicated the presence of the GppA enzyme in E. coli for the conversion of pppGpp to ppGpp. However, Hara and Sy (1983) were the first to purify, characterize, and show the substrate specificity of GppA enzyme of E. coli. Recently, Mechold et al. (2013) have successfully manipulated the expression of GppA to demonstrate differential accumulation of ppGpp and pppGpp in E. coli cells, which allowed them to conclude that pppGpp is less potent than ppGpp with respect to regulation of growth rate, RNA/DNA ratios, ribosomal RNA P1 promoter

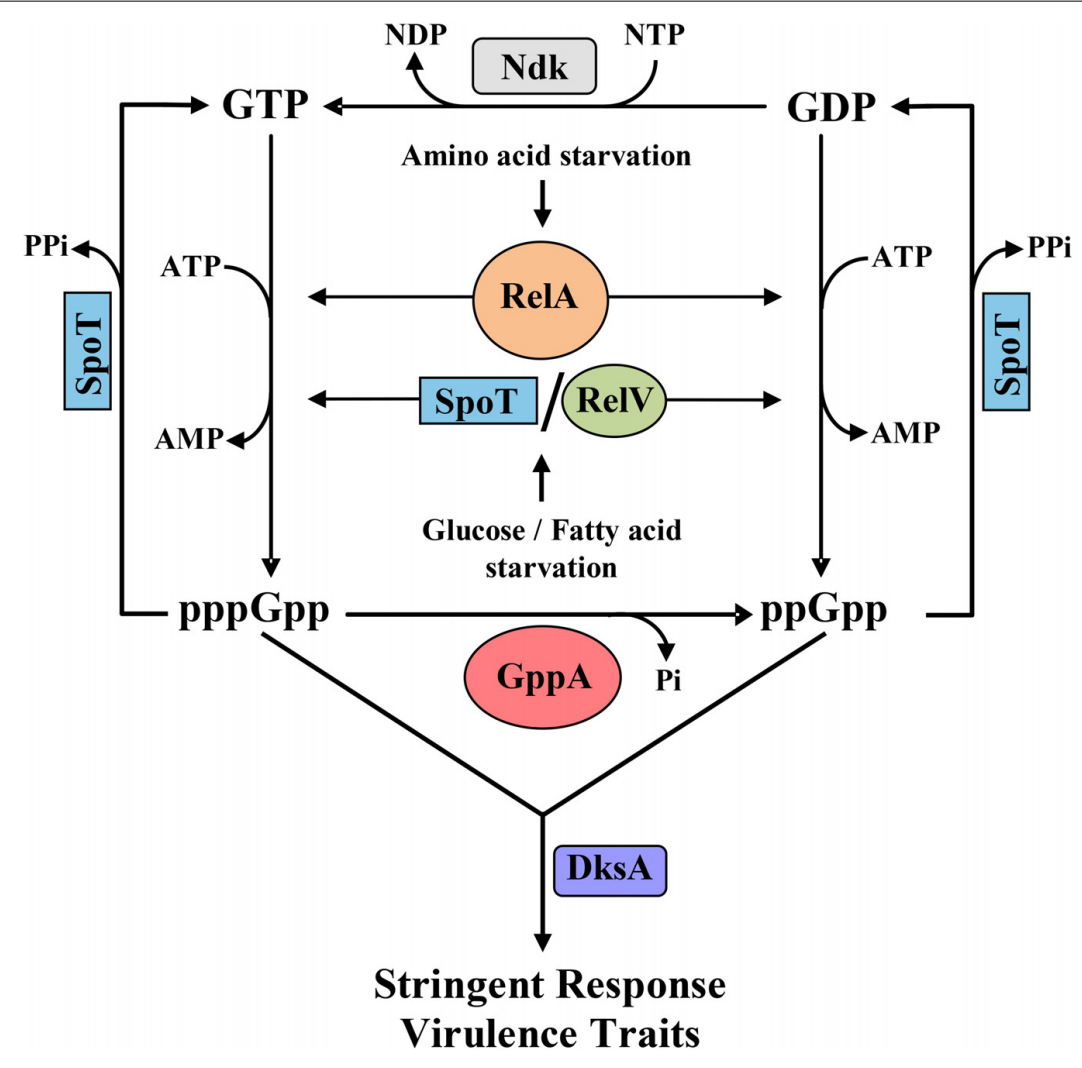

FIGURE 1 | Schematic diagram showing the stringent response (SR) metabolic cycle involving different enzymes of $V$. cholerae. As shown, the RelA enzyme is activated during amino acid starvation, while glucose or fatty acid starvation induces SpoT and RelV followed by synthesis of pppGpp and ppGpp using guanosine triphosphate (GTP) and guanosine diphosphate (GDP) as substrates, respectively. pppGpp is converted to ppGpp by the enzyme GppA (this study). SpoT, the bifunctional enzyme, has a strong hydrolase activity and thus hydrolyzes pppGpp and ppGpp to GTP and GDP, respectively. GDP is subsequently converted to GTP by the nucleotide diphosphate kinase (Ndk) enzyme. Synthesized pppGpp/ppGpp molecules with the help of the regulatory protein DksA controls the SR and several critical virulence traits of $V$. cholerae like cholera toxin production, biofilm formation, motility, and protease expression. 
transcription inhibition, threonine operon promoter activation, RpoS induction, etc. After the discovery of GppA, another phosphatase, called exopolyphosphatase (Ppx), was identified in E. coli (Akiyama et al., 1993). Ppx has been shown to hydrolyze the inorganic polyphosphate (polyP) to $\mathrm{P}_{i}$ (Akiyama et al., 1993). PolyP, a polymer of hundreds of phosphate residues, accumulates in $E$. coli and in other microbes in response to various stresses, including nutritional starvation (Kuroda et al., 2001). PolyP in E. coli and in other bacteria is synthesized by the enzyme polyphosphate kinase (Ppk), and the genes encoding Ppk and Ppx are usually physically linked (Akiyama et al., 1993; Kuroda et al., 1997, 2001; Ogawa et al., 2000). Extensive in silico analysis further indicated that GppA and $\mathrm{Ppx}$ are homologous proteins, and both of them belong to the sugar kinase/actin/hsp70 superfamily (Reizer et al., 1993; Koonin, 1994). It has been shown that like Ppx, the GppA enzyme of E. coli also has exopolyphosphatase activity, and thus, it is a bifunctional enzyme (Keasling et al., 1993). Similarly, a modest pppGpp hydrolase activity of E. coli Ppx has previously been demonstrated (Kuroda et al., 1997). It may be mentioned here that the members of Actinobacteria, like mycobacteria, corneybacteria, actinomycetes, etc., as well as certain other Gram-negative pathogens may encode homologous "Ppx-GppA" motif containing enzymes instead of separate Ppx and GppA proteins. The "Ppx-GppA" family of proteins share homology with both Ppx and GppA, and thus, they may have the ability to hydrolyze both polyP and pppGpp as substrates (Kristensen et al., 2004, 2008; Choi et al., 2012; Malde et al., 2014; Song et al., 2019).

Evolution of GppA supports that ppGpp is most likely the crucial molecule for the execution of SR in bacteria. Over the years, our group has characterized and established the entire SR regulatory genetic circuits including the discovery of the unique (p)ppGpp synthetase gene relV in $V$. cholerae as shown in Figure 1 (Haralalka et al., 2003; Das and Bhadra, 2008; Das et al., 2009; Pal et al., 2011, 2012; Dasgupta et al., 2014). In this (p)ppGpp metabolic cycle, conversion of GDP to GTP is carried out by the enzyme nucleoside diphosphate kinase, called $\mathrm{Ndk}$, which has been characterized by the other workers (Dar et al., 2011). We have shown that the SR regulatory circuit is also linked with the modulation of certain critical virulencerelated traits of $V$. cholerae (Haralalka et al., 2003; Pal et al., 2012; Basu et al., 2017; Basu and Bhadra, 2019). However, at present, no information is available about the functional aspect of the $g p p A$ gene in the cholera pathogen, the product of which converts pppGpp to ppGpp. Furthermore, very little information is available about the level of pppGpp and ppGpp under amino acid or glucose starved condition. Like in E. coli, $V$. cholerae genome also carries the physically linked $p p k-p p x$ genes, which are involved in polyP metabolism (Heidelberg et al., 2000; Ogawa et al., 2000). In this report, we have carried out extensive genetic analysis of the $g p p A$ gene and show that the product of the gene is linked with the (p)ppGpp metabolism in V. cholerae (Figure 1). Like in E. coli, Ppx is also homologous to GppA in V. cholerae; therefore, mutational approaches were adopted to analyze the function of $p p x$ in the presence or absence of the $g p p A$ gene. It was found that the overexpression of $V$. cholerae Ppx can complement the GppA function.
(Part of this work has been presented in the 54th United States-Japan Joint Panel Conference on Cholera and Other Bacterial Enteric Diseases held on 10-13 December 2019 at Osaka University, Osaka, Japan).

\section{MATERIALS AND METHODS}

\section{Bacterial Strains, Plasmids, Media, and Growth Conditions}

Bacterial strains and plasmids used in this study are described in Table 1. For cloning purpose, the plasmid pBluescript II $\mathrm{KS}(+)$ and $E$. coli $\mathrm{DH} 5 \alpha$ strain were used unless stated otherwise. Both $E$. coli and $V$. cholerae cells were routinely grown in Luria broth (LB; Difco, United States) at $37^{\circ} \mathrm{C}$ with aeration, and for plate culture, Luria agar (LA; Difco) was used, which contained $1.5 \%(\mathrm{w} / \mathrm{v})$ Bacto agar (Difco) as described earlier (Haralalka et al., 2003). Antibiotics (Sigma-Aldrich, United States) were used in the following concentrations: ampicillin (Ap), $100 \mu \mathrm{g}$ $\mathrm{ml}^{-1}$; kanamycin $(\mathrm{Km}), 40 \mu \mathrm{g} \mathrm{ml}^{-1}$; streptomycin $(\mathrm{Sm}), 100 \mu \mathrm{g}$ $\mathrm{ml}^{-1}$; chloramphenicol $(\mathrm{Cm}), 3 \mu \mathrm{g} \mathrm{ml}^{-1}$ for V. cholerae; and $30 \mu \mathrm{g} \mathrm{ml}^{-1}$ for E. coli. Bacterial strains were stored at $-70^{\circ} \mathrm{C}$ in LB containing 20\% (v/v) sterile glycerol (Verma et al., 2019) and were always taken out before doing experiments to avoid the development of any uncharacterized mutation. The growth of bacterial cultures was monitored by measuring optical density at $600 \mathrm{~nm}\left(\mathrm{OD}_{600}\right)$ using a spectrophotometer (Hitachi, Model U-5100).

\section{Molecular Biological Methods}

For chromosomal and plasmid DNA preparations, restriction enzyme digestion, DNA ligation, bacterial transformation, conjugation, agarose gel electrophoresis, etc., standard molecular biological methods (Ausubel et al., 1989) were followed. Restriction and nucleic-acid-modifying enzymes were purchased from New England Biolabs, Inc., United States and were used essentially as instructed by the manufacturer. Electrocompetent $V$. cholerae cells were made as described previously from this laboratory (Das and Bhadra, 2008), and transformants were selected on LA plates containing appropriate antibiotics.

\section{Construction of Plasmids and Mutants}

Throughout the study, the expression vector pBADcam (Table 1), a derivative of pBAD24 ( $4.5 \mathrm{~kb}$; $\mathrm{Ap}^{r}$; Table 1) carrying the $\mathrm{Cm}$ gene $(\mathrm{cam})$ cassette, was used. To construct pBADcam, the plasmid pPROTet.E (2.2 kb; $\mathrm{Cm}^{r}$; Table 1) was digested with $E c o \mathrm{RV}$, and $\sim 0.8 \mathrm{~kb}$ digested product carrying the cam cassette was ligated to ScaI-digested pBAD24; the ligation mixture was transformed into E. coli DH5 $\alpha$. Among multiple clones obtained, one was selected for use and named pBADcam $\left(\sim 5.3 \mathrm{~kb} ; \mathrm{Cm}^{r}\right.$; Table 1). Details of the primers used in this study are described in Supplementary Table S1. The recombinant plasmid pGppA (Table 1) was constructed by amplifying the gppA Open Reading Frame $(\mathrm{ORF})(\sim 1.6 \mathrm{~kb})$ using the primers gppAorf-F/gppAorf-R (Supplementary Table S1) and the genomic DNA of $V$. cholerae O1 El Tor strain N16961 [Table 1; henceforth will be called 
TABLE 1 | Bacterial strains and plasmids used in this study.

\begin{tabular}{|c|c|c|}
\hline Strains & Relevant genotype and/or phenotype & Source/reference \\
\hline \multicolumn{3}{|l|}{ V. cholerae } \\
\hline N16961 & Wild type, O1 serogroup, biotype El Tor, $\mathrm{Sm}^{r}$ & Lab stock \\
\hline $\mathrm{N} \Delta \mathrm{gAK}(\Delta g p p A)$ & N16961 $\Delta g p p A:: k a n ; \mathrm{Km}^{r} \mathrm{Sm}^{r}$ & This study \\
\hline $\mathrm{N} \Delta \mathrm{PPX}(\Delta p p x)$ & N16961 $\Delta p p x:: c a m ; \mathrm{Cm}^{r} \mathrm{Sm}^{r}$ & This study \\
\hline $\mathrm{N} \Delta \mathrm{gPx}(\Delta g p p A \Delta p p x)$ & $\mathrm{N} \Delta \mathrm{gAK} \Delta p p x:: c a m ; \mathrm{Cm}^{r} \mathrm{Km}^{r} \mathrm{Sm}^{r}$ & This study \\
\hline \multicolumn{3}{|l|}{ E. coli } \\
\hline $\mathrm{DH} 5 \alpha$ & F' endA1 hsdR17 supE44 thi-1 recA1 gyrA96 relA1 $\Delta(\operatorname{argF-lacZYA)~U169~(\Phi 80dlacZ\Delta M15)~}$ & Lab stock \\
\hline SM10גpir & thi thr leu tonA lacY supE recA::RP4-2-Tc::Mu $\lambda$ pir R6K & Lab stock \\
\hline \multicolumn{3}{|l|}{ Plasmids } \\
\hline pBluescript II KS(+) & ColE1, high-copy-number cloning vector; $\mathrm{Ap}^{r}$ & Stratagene \\
\hline pKAS32 & rpsL suicide vector with oriR6K mobRP4; $\mathrm{Ap}^{r}$ & Lab stock \\
\hline pBAD24 & pBR322 origin, L-arabinose inducible vector; $\mathrm{Ap}^{r}$ & Lab stock \\
\hline pROTet.E & ColE1 origin, high copy number expression vector; $\mathrm{Cm}^{r}$ & BD Biosciences \\
\hline pUC4K & Source of kan resistance gene cassette; $\mathrm{Ap}^{r} \mathrm{Km}^{r}$ & Pharmacia \\
\hline $\mathrm{pK} \Delta \mathrm{gAK}$ & 2.0-kb $\Delta g p p A:: k a n$ allele cloned in Kpnl/Sacl sites of pKAS32; $\mathrm{Ap}^{r} \mathrm{Km}^{r}$ & This study \\
\hline pKPXUDCam & 1.7-kb $\Delta p p x:: c a m$ allele cloned in Kpnl/Sacl sites of pKAS32; $\mathrm{Ap}^{r} \mathrm{Cm}^{r}$ & This study \\
\hline pBADcam & 0.8-kb cam resistance gene cassette from EcoRV digested pROTet.E was cloned in Scal site of pBAD24; Cm ${ }^{r}$ & This study \\
\hline pGppA & 1.6-kb gppA ORF of N16961 cloned in EcoRVPstl sites of pBADcam; Cmr & This study \\
\hline $\mathrm{pPpx}$ & 1.6-kb ppx ORF of N16961 cloned in Xbal/Hindlll sites of pBADcam; Cmr & This study \\
\hline pGppA1-272 & pGppA with 678-bp deletion from $3^{\prime}$-end of gppA ORF; $\mathrm{Cm}^{r}$ & This study \\
\hline pGppA1-303 & pGppA with 585-bp deletion from $3^{\prime}$-end of gppA ORF; $\mathrm{Cm}^{r}$ & This study \\
\hline pGppA1-308 & pGppA with 570-bp deletion from $3^{\prime}$-end of gppA ORF; $\mathrm{Cm}^{r}$ & This study \\
\hline pGppA1-310 & pGppA with 564-bp deletion from $3^{\prime}$-end of gppA ORF; $\mathrm{Cm}^{r}$ & This study \\
\hline pGppA1-313 & pGppA with 555-bp deletion from $3^{\prime}$-end of gppA ORF; $\mathrm{Cm}^{r}$ & This study \\
\hline pGppA13-497 & pGppA with 36-bp deletion from 5'-end of gppA ORF; $\mathrm{Cm}^{r}$ & This study \\
\hline pGppA18-497 & pGppA with 51-bp deletion from 5'-end of gppA ORF; $\mathrm{Cm}^{r}$ & This study \\
\hline pGppA20-497 & pGppA with 57-bp deletion from $5^{\prime}$-end of gppA ORF; $\mathrm{Cm}^{r}$ & This study \\
\hline pGppA23-497 & pGppA with 66-bp deletion from 5'-end of gppA ORF; $\mathrm{Cm}^{r}$ & This study \\
\hline pGppA18-310 & pGppA with 51-bp deletion from $5^{\prime}$-end and 564-bp deletion from $3^{\prime}$-end of gppA ORF; $\mathrm{Cm}^{r}$ & This study \\
\hline
\end{tabular}

Ap, ampicillin; Cm, chloramphenicol; Km, kanamycin; Sm, streptomycin.

wild type $(\mathrm{Wt})]$, followed by double digestion with the restriction enzymes EcoRI/PstI and cloning in similarly digested expression vector pBADcam. Similarly, the $p p x$ ORF $(\sim 1.6 \mathrm{~kb})$ carrying plasmid pPpx (Table 1) was constructed using the primers ppxORF-F/ppxORF-R (Supplementary Table S1) and genomic DNA of $V$. cholerae Wt strain. The PCR amplified $p p x$ ORF containing fragment was double digested with XbaI/HindIII and cloned in similarly digested vector pBADcam. For the construction of deletion mutants, we always used the suicide vector pKAS32 (Ap ${ }^{r}$; Table 1), which was maintained in E. coli SM10 $\lambda$ pir $\left(\mathrm{Km}^{r}\right.$; Table 1). Details of the recombinant suicide plasmids, $\mathrm{pK} \triangle \mathrm{gAK}$ and $\mathrm{pKPXUDCam}$, constructed in this study are given in Table 1. Apart from cam gene, the kan gene cassette from the plasmid pUC4K (Table 1) was used for creation of a deletion allele of a desired gene of $V$. cholerae. To construct any deletion mutant of $V$. cholerae, the corresponding recombinant suicide plasmid carrying the mutant allele was conjugally transferred from E. coli SM10 $\lambda$ pir to $V$. cholerae cells, and the desired deletion mutant was selected by double crossover event using appropriate antibiotics as described previously (Haralalka et al., 2003; Das et al., 2009). Details of the deletion mutants of $V$. cholerae constructed in this study are given in Table 1.
For construction of plasmids carrying amino (N)- and/or carboxy (C)-terminal coding region deleted $g p p A$ gene, each of such desired fragments was PCR amplified using specific set of primers (Supplementary Table S1) and genomic DNA of $V$. cholerae Wt strain as templates followed by digestion of the desired fragment with EcoRI/PstI and cloning of that DNA fragment in similarly digested vector DNA pBADcam. For amplification of $5^{\prime}$-end deleted fragment of $g p p A$ ORF each of the forward primers (Supplementary Table S1) always carried an artificially inserted ATG start codon. Similarly, for the amplification of $3^{\prime}$-end deleted fragment of the gppA ORF, each of the reverse primers (Supplementary Table S1) always carried an artificially inserted TAA stop codon. Restriction digestion and DNA sequencing further confirmed the recombinant plasmids.

\section{Detection of Intracellular (p)ppGpp Accumulation}

Intracellular accumulation of (p)ppGpp in $V$. cholerae cells under various starvation conditions was determined by radiolabeling of bacterial cells with $\left[{ }^{32} \mathrm{P}\right]-\mathrm{H}_{3} \mathrm{PO}_{4}$ (BRIT, Mumbai, India) using 3-(N-morpholino) propanesulfonic acid (MOPS) medium with different supplements followed by lysis of cells and thin 
layer chromatography (TLC) essentially as described previously (Haralalka et al., 2003; Das and Bhadra, 2008; Das et al., 2009). For (p)ppGpp labeling under no starvation conditions, the same MOPS medium mentioned above was used, but it contained all amino acids plus glucose. Bacterial cells were labeled as mentioned earlier (Haralalka et al., 2003; Das and Bhadra, 2008; Das et al., 2009) followed by autoradiography. Densitometric analysis of (p)ppGpp spots on autoradiogram was done using the ImageJ software ${ }^{1}$. Abundance of pppGpp in each lane relative to the total (pppGpp + ppGpp) was calculated by dividing the spot intensity of pppGpp with that of total intensity value of pppGpp + ppGpp.

\section{Reverse Transcriptase PCR Assay}

For reverse transcriptase PCR (RT-PCR) assay, $V$. cholerae cells were grown in $\mathrm{LB}$ at an $\mathrm{OD}_{600}$ value of $\sim 1.0$ followed by extraction of total cellular RNA using TRI Reagent (SigmaAldrich, United States) essentially as described previously (Pal et al., 2012; Basu et al., 2017; Basu and Bhadra, 2019). Purity and quantitation of prepared RNA were done spectrophotometrically. RT-PCR assay was carried out using the Qiagen One Step RTPCR kit as directed by the manufacturer (Qiagen, Germany). The PCR-amplified product was checked by agarose gel electrophoresis using appropriate molecular size markers. To confirm the absence of any contaminating DNA in prepared RNA samples, PCR assay of each sample was also done with Taq DNA polymerase (Invitrogen). Lack of amplification in the absence of RT confirmed that the desired PCR product was generated only from complementary DNA (cDNA). For quantitative RTPCR (qRT-PCR) assay, total cellular RNA was prepared from bacterial cells grown in $\mathrm{LB}$ medium to an $\mathrm{OD}_{600}$ value of $\sim 0.5$, 1.0 , or 1.5 as mentioned above. Reactions were done using the One Step SYBR ${ }^{\circledR}$ PrimeScript ${ }^{\mathrm{TM}}$ RT-PCR Kit II essentially as described by the manufacturer (Takara Bio Inc., Japan). qRTPCR assay was done using the CFX96 ${ }^{\mathrm{TM}}$ Real-Time System (Bio-Rad, United States). The primer set gppArtm-F/gppArtmR (Supplementary Table S1) was used for qRT-PCR analysis. Relative expression values $(R)$ were calculated using the equation $R=2^{-\left(\Delta C_{T} \text { target }-C_{T} \text { reference }\right)}$, where $C_{T}$ is the fractional threshold cycle. In each experiment, as an internal control, the recA-specific primers recA-F/recA-R (Supplementary Table S1) were used. Each assay was repeated at least thrice. To check the expression status of $g p p A$ during amino acid starvation, glucose starvation, and in the absence of any starvation, total cellular RNA was extracted from $V$. cholerae following the same experimental conditions as done for (p)ppGpp labeling experiment. The level of $g p p A$ transcripts was measured by qRTPCR analysis. As an internal control, the gyrA-specific primers, gyrArtm-F/gyrArtm-R (Supplementary Table S1), were used as recommended earlier (He et al., 2012). As positive controls, we used the $V$. cholerae biofilm related regulatory genes, $v p s R$ and $v p s T$, which have been shown to be upregulated during amino acid starvation (He et al., 2012). Each RT-PCR experiment was repeated at least thrice.

\footnotetext{
${ }^{1}$ https://imagej.nih.gov
}

\section{DNA Sequencing and Computational Analyses}

For confirmation of plasmid constructs, DNA sequencing was done using the BigDye ${ }^{\circledast}$ Terminator v3.1 Cycle Sequencing Kit (Applied Biosystems Inc., United States) essentially as instructed by the manufacturer. The samples were run on an ABI3130 Genetic Analyzer using the pop-7 polymer (Applied Biosystems Inc.). DNA sequence data were compiled and analyzed by using the Chromas $1.45^{2}$. DNA sequences were obtained from J. Craig Venter Institute (JCVI) ${ }^{3}$, and protein domain information was obtained from Kyoto Encyclopedia of Genes and Genomes (KEGG) ${ }^{4}$. BLASTN and BLASTP programs were used to search for homologous nucleotide or protein sequences, respectively, in the database ${ }^{5}$. Clustal Omega software ${ }^{6}$ was used for the alignment of protein sequences and further modified through GeneDoc software version 2.7.0007. For designing primers, Primer3 software ${ }^{8}$ was used. For designing qRT-PCR primers, Primer Express 3.0 software (Applied Biosystems, United States) was used.

\section{Statistical Analysis}

Where needed, pairwise comparison of data for each sample was analyzed for statistical significance using Student's $t$-test.

\section{RESULTS}

\section{In silico Analysis of the gppA Gene of V. cholerae}

Since no information is currently available about the $g p p A$ gene of $V$. cholerae, BLASTN ${ }^{9}$ analysis of the whole-genomesequenced $V$. cholerae Wt strain N16961 (Table 1) was carried out using the E. coli gppA gene sequence as a query. Such analysis indicated that the large chromosome of $V$. cholerae indeed carries a 1.5-kb long gppA gene (JCVI annotation no. VC0304). Comparison of the gppA gene sequence of $V$. cholerae indicated $63 \%$ nucleotide sequence identity with that of $E$. coli by using the BLAST Global Alignment Tool. While the $V$. cholerae gPpA gene codes for a 497 amino acid long protein, the E. coli GppA is composed of 494 amino acids (see footnote 3). BLASTP analysis of $V$. cholerae GppA protein shows $57 \%$ identity (279 out of 491 amino acids) and $72 \%$ similarity with that of $E$. coli GppA. Genetic organization of the gppA gene in the genomes of $V$. cholerae, other Vibrio spp., and E. coli indicated that the $g p p A$ locus is highly conserved in vibrios (Figure 2A). BioCyc analysis ${ }^{10}$ suggested that the $g p p A$ gene along with its upstream region is physically linked with the rhlB gene (JCVI annotation

\footnotetext{
${ }^{2}$ http://www.technelysium.com.au/chromas.html

${ }^{3}$ www.jcvi.org

${ }^{4}$ www.genome.jp/kegg/

${ }^{5}$ www.ncbi.nlm.nih.gov

${ }^{6}$ https://www.ebi.ac.uk/Tools/msa/clustalo/

${ }^{7}$ www.psc.edu/biomed/genedoc

${ }^{8}$ http://frodo.wi.mit.edu/

${ }^{9}$ https://blast.ncbi.nlm.nih.gov/blast.cgi

${ }^{10}$ http://biocyc.org/VCHO/
} 

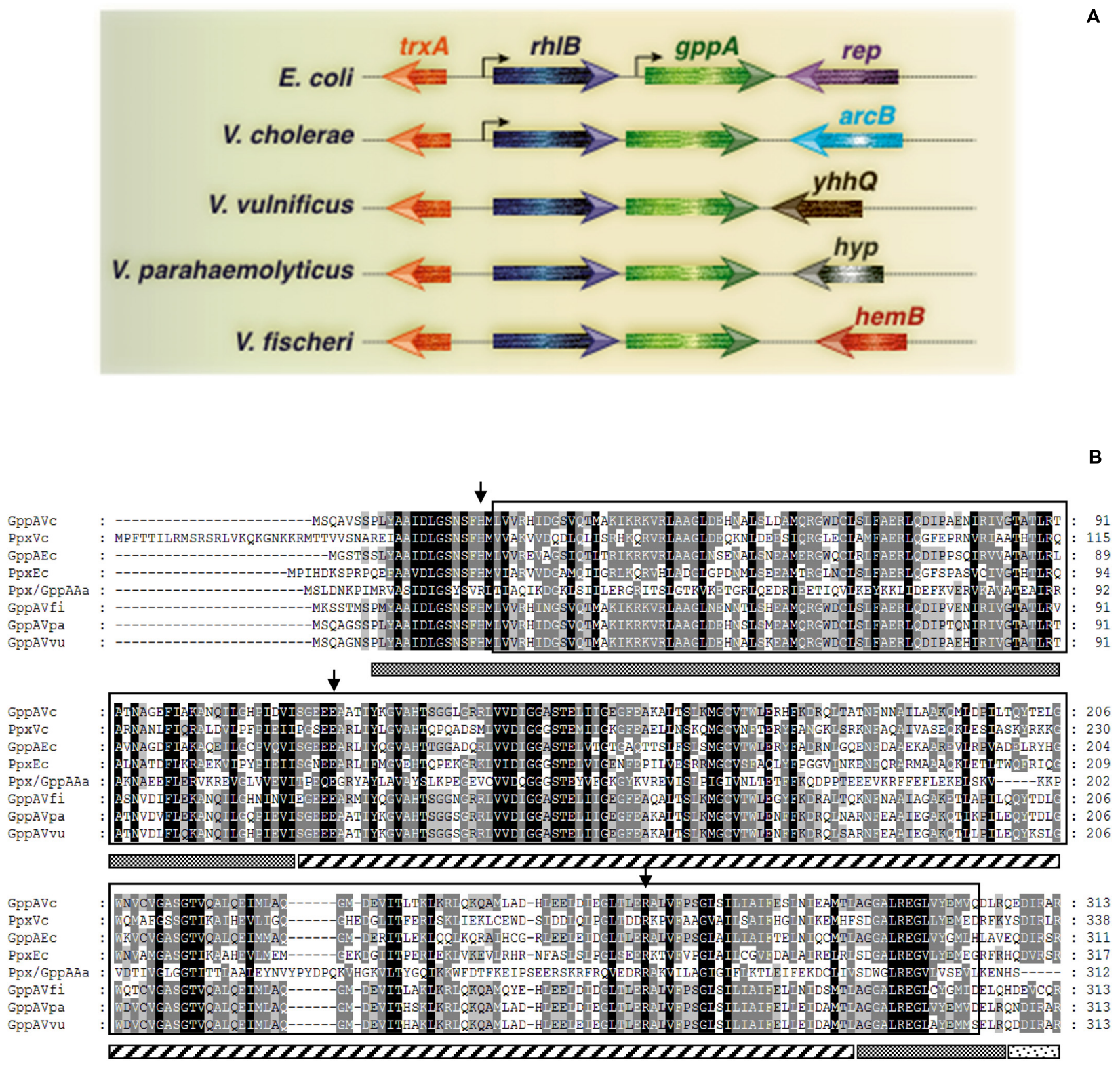

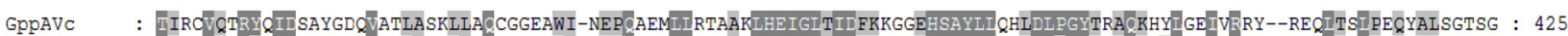

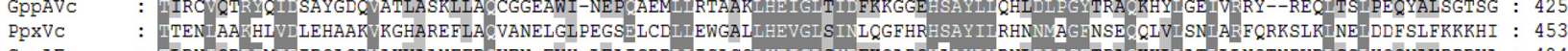

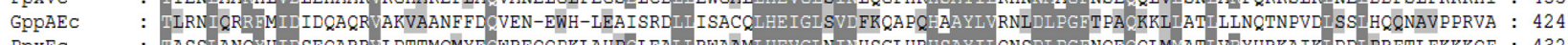
PpxEc $\mathrm{Ppx} / \mathrm{GppAAa}:$

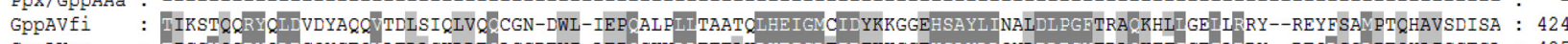

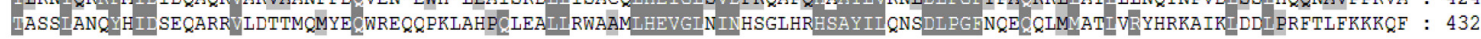

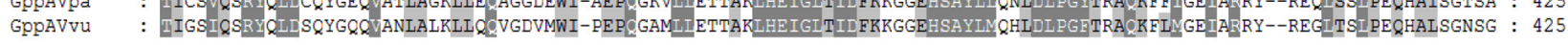

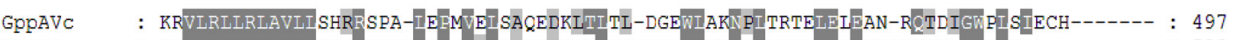
PpXVC : IGLIRVIRLA IVYNGQRNDDPI PE-ITISAKDDEWRIECEQPDWIENMKILHADIQTEQEYWREV-GW QIIF--------- : 523

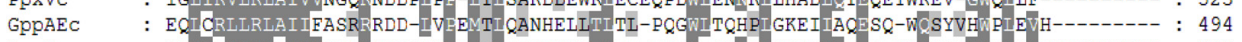

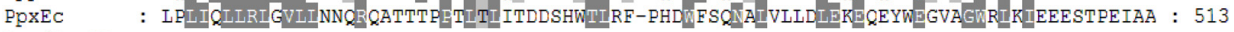

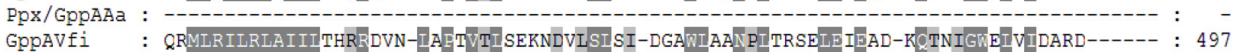

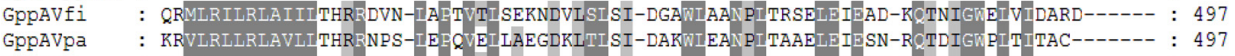

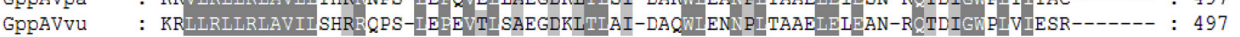

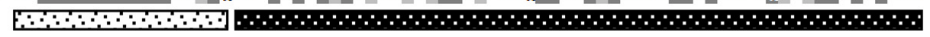

Domain I

Z2 Domain II

$\because$ Domain III

$\because$ Domain IV

FIGURE 2 | Continued 
FIGURE 2 | (A) Schematic diagram (not drawn to scale) showing genomic arrangement of the gppA gene in V. cholerae, E. coli, and other Vibrio spp. Names of different organisms are given in the left margin. Physically linked genes of gppA and their homologs are as indicated. Arrowheads indicate direction of transcription of genes. Thin gray line represents intergenic or chromosomal DNA. Bend arrows indicate putative monocistronic and bicistronic transcription of the gppA-rh/B genes in E. coli and V. cholerae, respectively. (B) Comparison of the amino acid sequences of Ppx-GppA motif containing proteins. Alignment was done using the Clustal Omega software (https://www.ebi.ac.uk/Tools/msa/clustalo/) and further modification by GeneDoc software version 2.7.000. Aligned proteins include GppA (GppAVc) and Ppx (PpxVc) of V. cholerae with those of E. coli GppA (GppAEc), E. coli Ppx (PpxEc), A. aeolicus Ppx/GppA (Ppx/GppAAa), V. fischeri GppA (GppAVfi), V. parahaemolyticus GppA (GppAVpa), and V. vulnificus GppA (GppAVvu). Dark and light shades indicate identical and similar amino acid, respectively. Boxed amino acid sequence denotes highly conserved Ppx-GppA motif (usually about 280 amino acids long). Small vertical arrows indicate conserved critical amino acid residues needed for phosphohydrolase activity. Four probable domains (domains I-IV) are indicated as different patterned bars. The domains are marked considering the PpxEc protein as a reference. Numbers in the right margin indicate amino acids length of each protein.

no. VC0305), and they transcribe as a bicistronic operon. KEGG analysis of the 497 amino acid long GppA protein of $V$. cholerae revealed that it carries a highly conserved Ppx-GppA motif of 281 amino acids long (position 23 to 303), which is similar to the Ppx-GppA motif of E. coli GppA enzyme (Figure 2B). We also did BLASTN analysis for the $V$. cholerae $p p x$ gene (JCVI annotation no. VC0722) and found that it encodes 523 amino acids long exopolyphosphatase. BLASTP analysis indicated that the $V$. cholerae Ppx has $40.5 \%$ identity with that of GppA (Figure 2B). Furthermore, amino acid sequence alignment of the GppA and Ppx proteins of $V$. cholerae, other Vibrio spp., E. coli, and thermophilic Aquifex aeolicus indicated substantial conservation of the residues including those needed for the pppGpp hydrolysis function (see Figure 2B). Analyses of the crystal structures of the E. coli $\mathrm{Ppx}$ and GppA proteins indicated the presence of four distinct domains, I-IV (Rangarajan et al., 2006; Song et al., 2019). It is evident that the V. cholerae Ppx and GppA proteins also carry similar domains as shown in Figure 2B.

\section{Transcriptional Organization of the gppA Operon of $V$. cholerae}

Comparison of the gppA genetic loci of $V$. cholerae and E. coli revealed distinct difference in organization of different ORFs (Figure 2A). Bioinformatic analysis indicated that the $r h l B$ $g p p A$ genes of $V$. cholerae are arranged in a bicistronic operon since there is only a 7-bp gap between these two genes. The transcription orientations of the flanking ORFs of the $r h l B-g p p A$ operon are in reverse orientations (Figure 2A). In sharp contrast, the E. coli $g p p A$ gene appears to be organized as a monocistronic transcription unit since there is a 135 -bp long intergenic region with predicted promoter sequence between the $r h l B$ and $g p p A$ genes (Figure 2A). While the downstream region of gppA of $V$. cholerae contains the $\operatorname{arc} B$ gene encoding a sensor histidine kinase, in E. coli, the rep gene encoding an ATP-dependent DNA helicase is present in the same position (Figure 2A). In this study, we predicted that the $V$. cholerae $g p p A$ gene (JCVI annotation no. VC304) cotranscribes with the $r h l B$ gene (JCVI annotation no. VC0305), and they are arranged in a bicistronic operon. To confirm this, $V$. cholerae cells were grown till late exponential phase $\left(\mathrm{OD}_{600}=1.0\right)$, and total cellular RNAs were prepared for RT-PCR analysis. When the primer pair gppASeq-F (VC0305 ORF specific) and gppArtm-R (Supplementary Table S1) was used, as expected, a desired cDNA fragment of $\sim 0.33 \mathrm{~kb}$ in size was obtained (Figure 3). Similarly, when gppA or VC0305 ORF specific internal primers (gppArtm-F/gppA-R or rhlB-F/rhlB-R;

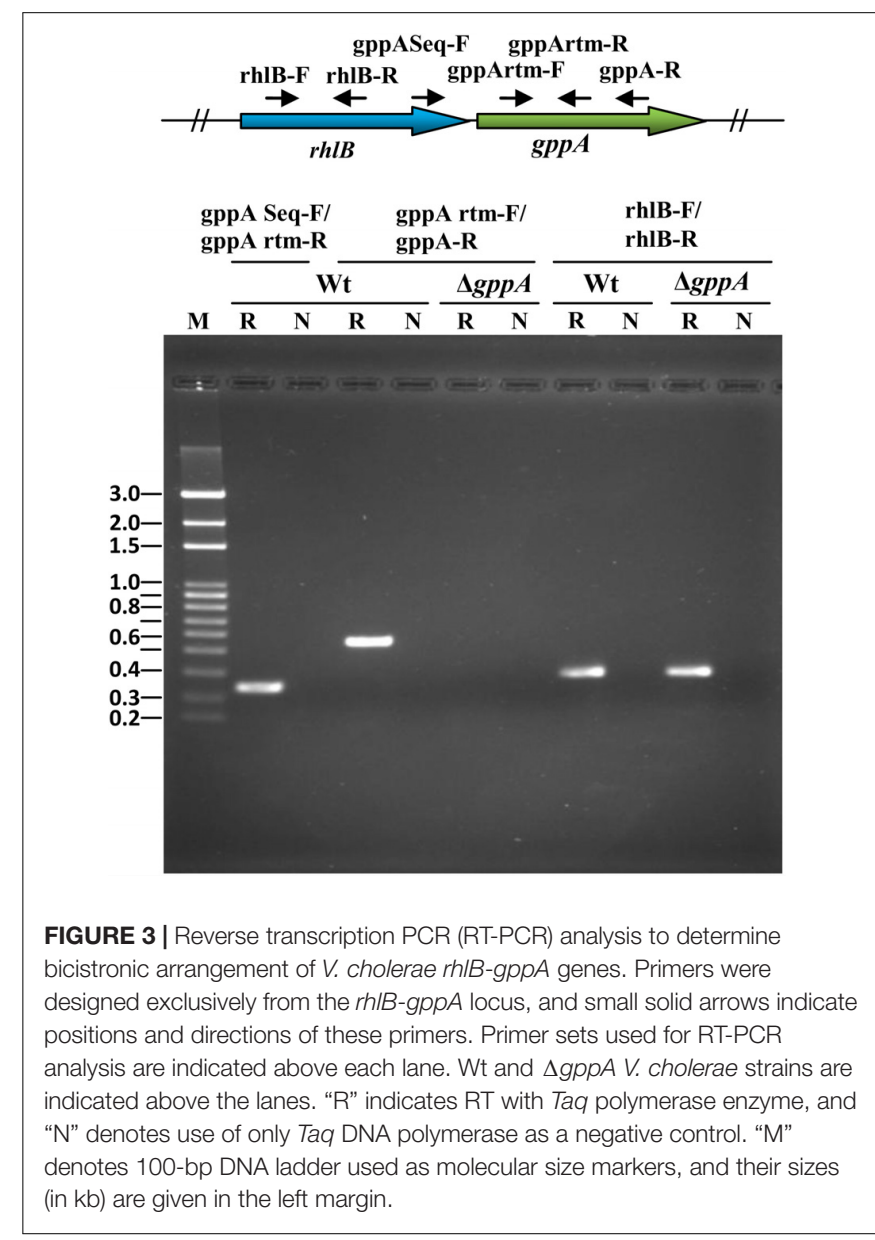

Supplementary Table S1) were used, in each case, the desired cDNA fragment of size $\sim 0.56$ or $0.37 \mathrm{~kb}$, respectively, was obtained (Figure 3). The RT-PCR results confirmed that the gppA and VC0305 $(r h l B)$ genes are indeed arranged in an operon, and they are cotranscribed. Next, we wanted to ensure that in the gppA-deleted $V$. cholerae strain N $\triangle \mathrm{gAK}$ (Table 1; henceforth will be called $\triangle g p p A$ ), the transcription of the neighboring gene $r h l B$ (VC0305) was not affected. It is to be noted that during construction of the $\triangle g p p A$ mutant, the closest $r h l B$ ORF was kept intact; however, transcription of the $\operatorname{arc} B$ gene was not tested. For this purpose, the transcript status of $r h l B$ in $\triangle g p p A$ strain was examined by RT-PCR using the same set of primer rhlB-F/rhlBR (Supplementary Table S1). While no cDNA was detectable for 
the gppA gene, $r h l B$ ORF-specific primer set was indeed able to amplify the desired cDNA fragment of $\sim 0.37 \mathrm{~kb}$ using the $\triangle g p p A$ mutant (Figure 3).

\section{GppA Modulates (p)ppGpp Homeostasis During Amino Acid Starvation}

In order to understand the functions of GppA in regulating the intracellular pppGpp and ppGpp levels in $V$. cholerae under various starved conditions, intracellular (p)ppGpp labeling assay was performed using the $\triangle g p p A$ mutant (Table 1) and its parental Wt strain. Before performing different experiments with the $\triangle g p p A$ mutant, its growth kinetics in LB and M9 minimal medium were compared with that of Wt cells. However, in both the media, $\triangle g p p A$ mutant showed no defect in growth when compared with the Wt (Supplementary Figures S1A,B). We have shown previously that the RelA of $V$. cholerae exclusively produces ppGpp under amino acid starved condition (Haralalka et al., 2003; Das and Bhadra, 2008). It may be argued here that under amino acid starvation, $V$. cholerae RelA may use GDP as a major substrate to synthesize ppGpp or it could use GTP as a substrate to produce pppGpp, which is rapidly converted to ppGpp by GppA. Interestingly, when $\triangle g p p A$ cells were starved for amino acids along with its isogenic Wt strain and labeled with ${ }^{32} \mathrm{P}$ followed by autoradiography, a significant increase $(\sim 10$-fold, see Figure 4$)$ in the intracellular concentration of pppGpp in $\triangle g p p A$ mutant was observed compared to the Wt (Figure 4A). The result suggests that, under amino acid starvation, GTP is also used as a substrate by the $V$. cholerae RelA to produce pppGpp followed by conversion to ppGpp by GppA. But in the case of $\triangle g p p A$ mutant, which is devoid of GppA, this conversion is not possible, and thus, the mutant cells showed accumulation of substantial amount of pppGpp. This observation is also supported by densitometric analysis of the (p)ppGpp spots using the ImageJ software as shown in Figure $4 \mathrm{~B}$. This is further supported by the complementation assay using the $\triangle g p p A$ cells carrying the $V$. cholerae $g p p A$ gene expressing plasmid pGppA (Table 1). As expected, such an assay and (p)ppGpp spot quantification clearly showed significant accumulation of ppGpp in $\triangle g p p A(\mathrm{pGppA})$ cells like that of the parental Wt strain (Figures $4 \mathrm{D}, \mathrm{E}$ ). It is important to mention here that functional complementation by GppA was also observed in the case of $\triangle g p p A(\mathrm{pGppA})$ in the absence of arabinose (Figure 4D), probably due to the leaky expression of $g p p A$ from the plasmid pGppA. By comparison of the profiles of pppGpp and ppGpp under amino acid starvation in $V$. cholerae relA $A^{+} g p p A^{+}(\mathrm{Wt})$ and $r e l A^{+} g p p A^{-}(\triangle g p p A)$ genetic backgrounds, it appears that the GDP is a more preferred substrate for RelA than GTP (Figure 4A). In support of this conclusion, it may be mentioned here that the E. coli RelA is able to synthesize pppGpp and ppGpp using GTP and GDP as substrates, respectively (Sajish et al., 2009; Kudrin et al., 2018). It has been shown that pppGpp allosterically activates RelA leading to its increased efficiency in the synthesis of ppGpp from GDP (Kudrin et al., 2018). Thus, through all these genetic analyses, we first time established the function of GppA in V. cholerae.

\section{Overexpression of Ppx Complements $\Delta g p p A$ Mutant During Amino Acid Starvation}

Since the $p p x$ gene of $V$. cholerae showed high homology with the $g p p A$ gene (Figure 2B), we also constructed $V$. cholerae $p p x$ and $g p p A p p x$ deletion mutants to test the pppGpp level during amino acid starvation in the presence and absence of functional $p p x$ gene. $V$. cholerae $p p x$ and $g p p A p p x$ mutants constructed for this purpose were designated as $\mathrm{N} \triangle \mathrm{PPX}$ (hereafter will be called $\triangle p p x$ ) and $\mathrm{N} \Delta \mathrm{gPx}$ (henceforth will be designated $\triangle g p p A \triangle p p x$ ), respectively (Table 1). While $\Delta p p x$ behaved like $\mathrm{Wt}$, the $\triangle g p p A \Delta p p x$ double mutant accumulated similar amount of pppGpp like in $\triangle g p p A$ cells (Figure 4A). This was further verified by densitometric quantification of the (p)ppGpp spots (Figure 4B). To confirm that the spots shown in Figure 4A are indeed of (p)ppGpp, as a control experiment, the same set of bacterial strains was used to label (p)ppGpp using ${ }^{32} \mathrm{P}$ without inducing any starvation. As expected, there was no accumulation of pppGpp and ppGpp in nutrient supplemented strains compared to the amino-acid-starved Wt and $\triangle g p p A$ cells (Figure 4C). Apparently, the result suggests that the Ppx enzyme is probably unable to convert pppGpp to ppGpp, which could be due to its low expression/activity under the experimental condition used. To test this hypothesis, we have constructed a Ppx-overexpressing plasmid pPpx (Table 1). When Ppx was overexpressed in $\triangle g p p A$ cells through the recombinant plasmid pPpx, it was able to complement the GppA function (Figure 4F). Densitometric analysis of the (p)ppGpp spots also supported this result as shown in Figure 4G. The result suggests that, under natural physiological condition, the basal level of Ppx probably has no or very weak pppGpp to ppGpp conversion activity. However, high abundance of the Ppx protein achieved by overexpression in $\triangle g p p A$ cells most probably helped in overcoming this defect and converted pppGpp to ppGpp. The result warrants further studies to elucidate the exact role of Ppx in the metabolism of (p)ppGpp in V. cholerae under different nutritional stress conditions.

\section{GppA Mediated Conversion of pppGpp to ppGpp Is Not Observed During Glucose Starvation Condition}

We further examined the status of pppGpp and ppGpp in $V$. cholerae $\triangle \mathrm{g} p p A$ mutant under glucose starvation. Interestingly, there was no difference in intracellular pppGpp and ppGpp levels between $\triangle g p p A$ and isogenic Wt cells (Figure 5A). We extended our analysis to test the pppGpp and ppGpp levels in $V$. cholerae $\triangle g p p A, \Delta p p x$, and $\triangle p p x \Delta g p p A$ mutants. As expected, no difference was observed in the intracellular pppGpp and ppGpp levels among $\mathrm{Wt}, \triangle g p p A, \Delta p p x$, and $\Delta g p p A \Delta p p x$ mutants either in the autoradiogram or by densitometric analysis (Figures 5A,B). At present, the mechanism(s) underscoring this contrasting function of $V$. cholerae GppA under amino-acid- and glucose-starved conditions is not clear. The possibility of the presence of interacting partner(s) that modulate GppA activity during glucose starvation or differential expression pattern of 

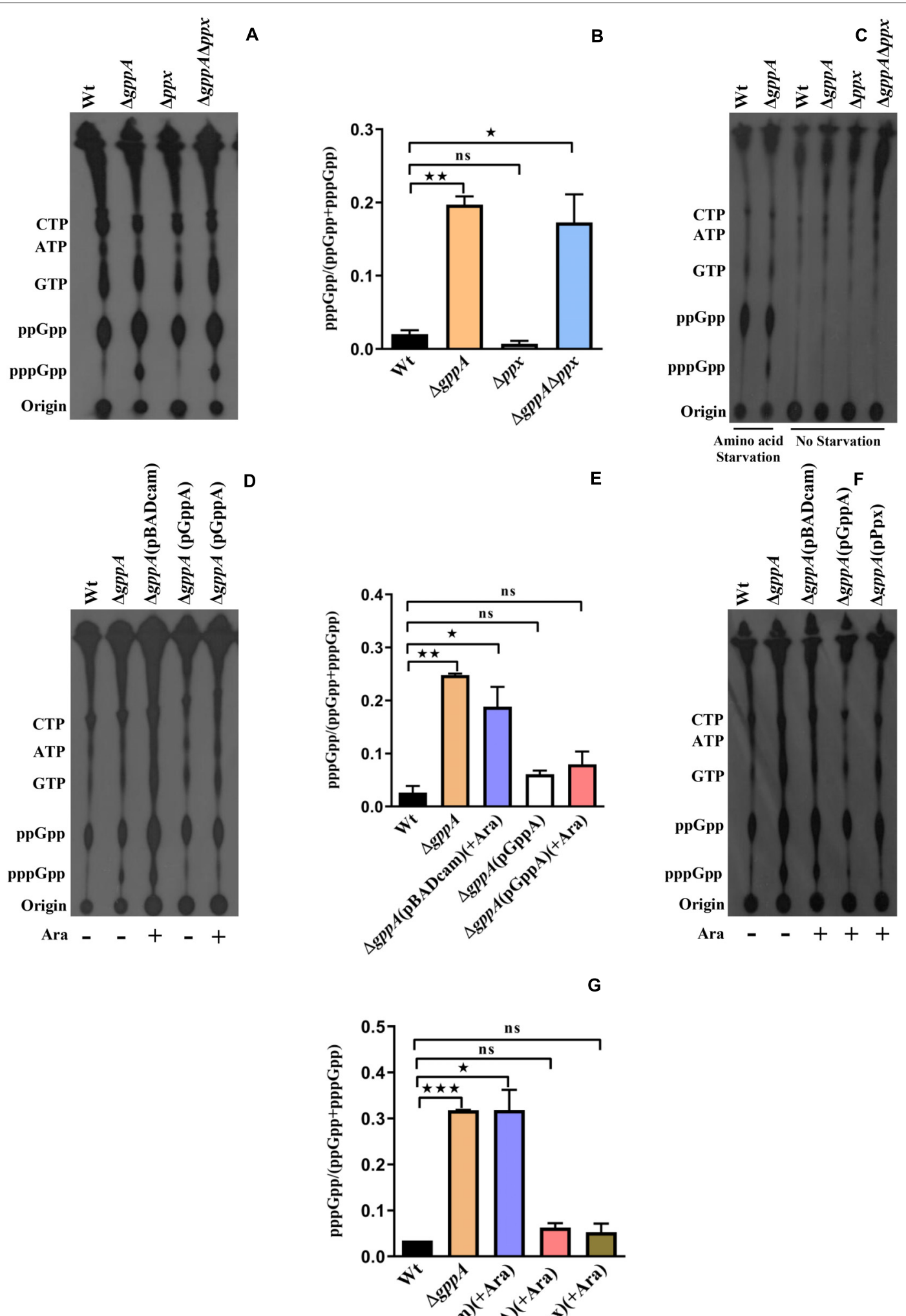
FIGURE 4 | (A) Accumulation of (p)ppGpp in V. cholerae cells under amino-acid-starved condition. Bacterial cells were labeled with ${ }^{32}$ P-orthophosphoric acid, extracted, resolved by TLC followed by autoradiography. Strains used are Wt, $\Delta g p p A, \Delta p p x$, and $\Delta g p p A \Delta p p x$. Distinct accumulation of pppGpp in $\Delta g p p A$ is evident. The presence of other labeled nucleotides including pppGpp/ppGpp is indicated in the left margin. (B) Densitometry of the (p)ppGpp spots detected in the autoradiogram was carried out by ImageJ software, and the ratio of pppGpp/(pppGpp + ppGpp) was plotted. Values represent the average of two independent experiments $(n=2)$, and one representative autoradiogram is shown in (A). Error bars indicate standard deviation. $\left({ }^{\star} P<0.05 ;{ }^{\star \star} P<0.01 ; \mathrm{ns}, \mathrm{non}-\mathrm{significant)}\right.$. (C) Accumulation of (p)ppGpp in $V$. cholerae cells without any starvation (see details in text). Strains used are Wt, $\Delta g p p A, \Delta p p x$, and $\Delta g p p A \Delta p p x$. (p)ppGpp accumulation in Wt and $\triangle g p p A$ under amino acid starvation condition served as controls. The presence of ${ }^{32} \mathrm{P}$-labeled nucleotides is indicated in the left margin. (D) Complementation analysis of the $\triangle g p p A$ mutant of $V$. cholerae. Autoradiogram shows poor or no accumulation of pppGpp in $\triangle g p p A(p G p p A)$ strain in which gppA was expressed through the plasmid pGppA using $0.2 \%$ arabinose (Ara) as an inducer. Wt, $\triangle g p p A$, and $\triangle g p p A$ carrying the empty plasmid $[\triangle g p p A(p B A D c a m)]$ strains were used as controls. The presence of labeled nucleotides including pppGpp/ppGpp as indicated in the left margin. (E) Densitometric analysis of the (p)ppGpp spots was done as described in (B). Values represent the average of two independent experiments $(n=2)$, and one representative autoradiogram is shown in (D). Error bars indicate standard deviation. ( ${ }^{\star} P<0.05$; ${ }^{\star \star} P<0.01$; ns, non-significant). (F) Autoradiogram showing (p)ppGpp accumulation in $\triangle g p p A$ mutant by overexpressing $p p x$. Induction of both gppA and $p p x$ expression through the plasmids pGppA and pPpx, respectively, was done using $0.2 \%$ arabinose as an inducer. Controls used are as given in (D). The presence of labeled nucleotides including pppGpp/ppGpp as indicated in the left margin. (G) Densitometric analysis of the (p)ppGpp spots was done as described in (B). Values represent the average of two independent experiments ( $n=2)$, and one representative autoradiogram is shown in (F). Error bars indicate standard deviation. $\left({ }^{\star} P<0.05\right.$; ${ }^{\star \star \star} P<0.001$; ns, non-significant).
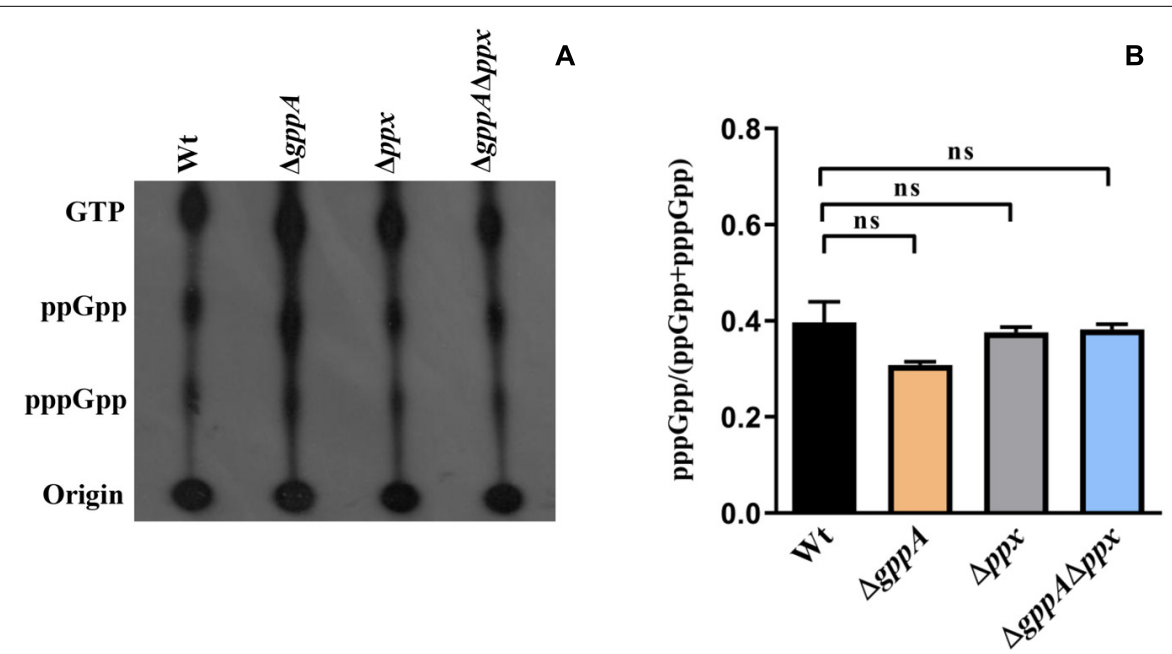

FIGURE 5 | (A) Accumulation of (p)ppGpp in V. cholerae cells under glucose starvation. Strains used are: Wt, $\Delta g p p A, \Delta p p x$, and $\Delta g p p A \Delta p p x$. The presence of labeled nucleotides including pppGpp/ppGpp is indicated in the left margin. (B) Densitometry of the (p)ppGpp spots detected in the autoradiogram was carried out by ImageJ software and the ratio of pppGpp/(pppGpp + ppGpp) was plotted. Values represent the average of two independent experiments $(n=2)$ and one representative autoradiogram is shown in Panel A. Error bars indicate standard deviation. (ns = non-significant).

gppA during glucose and amino acid starvations cannot be ruled out and needs further investigation.

\section{Expression of the gppA Gene Increases During Stationary Growth Phase and Amino Acid Starvation Condition}

After establishing the function of $g p p A$ gene in amino-acidstarved cells, we attempted to test its regulation, if any, during various growth phases and starvation conditions. To this end, we performed quantitative estimation of the $g p p A$ gene transcripts under various growth phases of $V$. cholerae cells in nutrient-rich LB medium using qRT-PCR assay. As shown in Figure $6 \mathrm{~A}$, there was significant increase $(\sim 2.5$-fold $)$ in $g p p A$ transcripts level in stationary phase cells of $V$. cholerae $\mathrm{Wt}$ strain compared to that of early or mid-log phase cells. We further extended our analysis to explore the expression level of the gppA gene in $V$. cholerae under both amino acid as well as glucose-starved conditions with respect to non-starved condition in MOPS medium using qRT-PCR assay (see details in section "Materials and Methods"). Interestingly, qRT-PCR analysis indicated a significant downregulation $(\sim 1.8$-fold $)$ of $g p p A$ expression in glucose-starved $V$. cholerae cells compared to non-starved bacteria (Figure 6B). On the other hand, it has been observed that $g p p A$ expression goes up ( $\sim 2.5$-fold) during amino acid starvation compared to non-starved cells (Figure 6B). When we compared the $g p p A$ expression between glucose and amino acid starved cells, a significant $(\sim 4.5$-fold) upregulation was observed in the latter condition (Figure 6B). This differential expression pattern of $g p p A$ during amino acid and glucose starvations may be responsible for the lower level of pppGpp accumulation in Wt $V$. cholerae cells under amino acid starvation. To support this observation, as positive controls, we measured the expression levels of two biofilm regulatory genes $v p s R$ and $v p s T$ of $V$. cholerae. It has previously been reported that the $v p s R$ and $v p s T$ genes are upregulated during amino acid starvation conditions (He et al., 2012). Therefore, we compared the expression of $g p p A, v p s T$, and $v p s R$ in amino-acid-starved 
LB medium

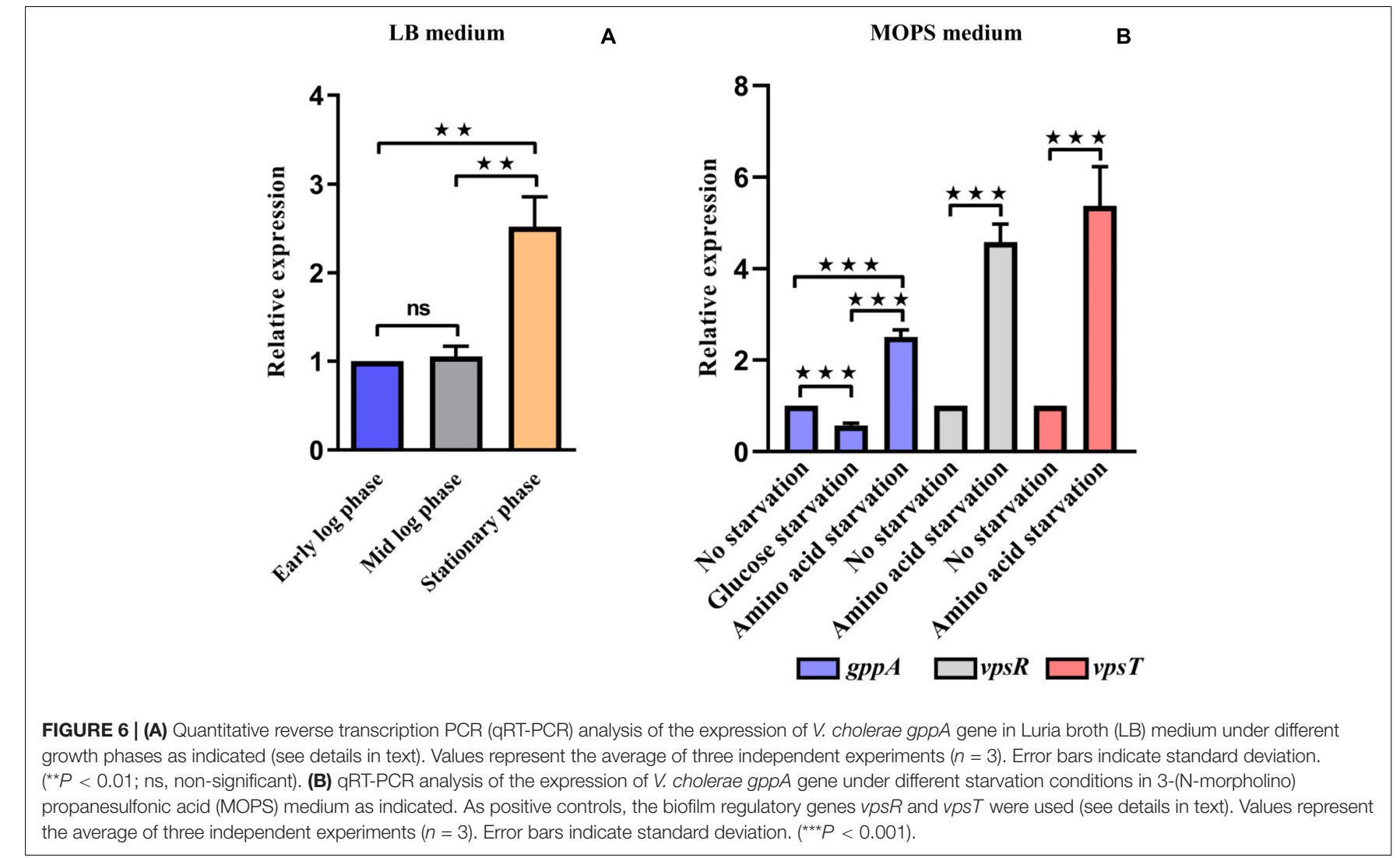

A

MOPS medium

B
$V$. cholerae cells. As expected, the expression of both $v p s R$ and $v p s T$ genes are indeed upregulated ( $\sim 5$-fold) during aminoacid-starved condition compared to non-starved $V$. cholerae cells (Figure 6B).

\section{Flanking Sequences Surrounding the Ppx-GppA Motif of GppA Are Important for Its Function}

To determine the importance of the $\mathrm{N}$ - and $\mathrm{C}$-terminal regions as well as the conserved domains (I-IV) of the $V$. cholerae GppA protein in pppGpp to ppGpp conversion function, several progressive deletion constructs (Figure 7A) were made by cloning each of the truncated $g p p A$ ORF under the $\mathrm{P}_{B A D}$ promoter of the plasmid pBADcam (Table 1), and each recombinant clone was used for complementation in $V$. cholerae $\triangle g p p A$ strain by assaying the ability of the expressed mutant alleles in intracellular conversion of pppGpp to ppGpp during amino acid starvation. While the $\triangle g p p A$ mutant strains carrying the plasmid pGppA1-313 [ $\triangle g p p A(\mathrm{pGppA} 1-$

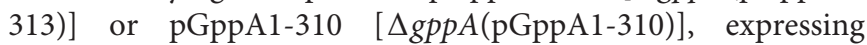
truncated GppA where 184 or 187 amino acids were deleted, respectively, were able to complement the GppA function, the strain $\triangle g p p A(\mathrm{pGppA1-272),} \triangle g p p A(\mathrm{pGppA1-303),} \mathrm{or}$ $\triangle g p p A$ (pBG1-308), where truncated $g p p A$ expressed 225, 194, or 189 C-terminally amino acids deleted proteins, respectively, failed to complement (Figure 7B). On the other hand, the $\triangle g p p A$ mutant strains carrying the plasmid pGppA13-497 $[\triangle g p p A(\mathrm{pGppA} 13-497)]$ or pGppA18-497 [ $g p p A(\mathrm{pGppA18-}$ 497)], expressing N-terminally 12 or 17 amino acids deleted proteins, were able to complement the GppA function but not

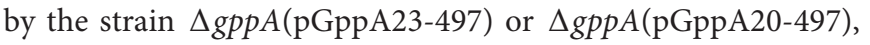
expressing $\mathrm{N}$-terminally 22 or 19 amino acids deleted GppA, respectively (Figure 7C). Finally, based on the above results, a recombinant plasmid pGppA18-310 (Table 1) containing the truncated $g p p A$ gene was constructed (Figure 7A), which should express $\mathrm{N}$-terminally (17 amino acids) and C-terminally (187 amino acids) deleted GppA protein. As expected, when the plasmid pGppA18-310 was introduced into the $\triangle g p p A$ mutant, it was able to complement the GppA function (Figure 7D). Thus, it appears that the functional $\mathrm{N}$ - and C-terminal boundary of GppA resides between the amino acid positions 18 and 310. In fact, the conserved Ppx-GppA motif actually falls within this minimal functional region of the protein (Figure 2B). To our knowledge this is the first report about functional boundary determination of a bacterial GppA protein.

\section{Effect of Deletion of the gppA Gene on Virulence Phenotypes of $V$. cholerae}

We have also examined the effect of deletion of the gppA gene on certain virulence phenotypes of $V$. cholerae like cholera toxin production, biofilm formation, and motility. However, no defect in any of these virulence phenotypes was observed in the $\triangle g p p A$ mutant (Supplementary Figure S2). 


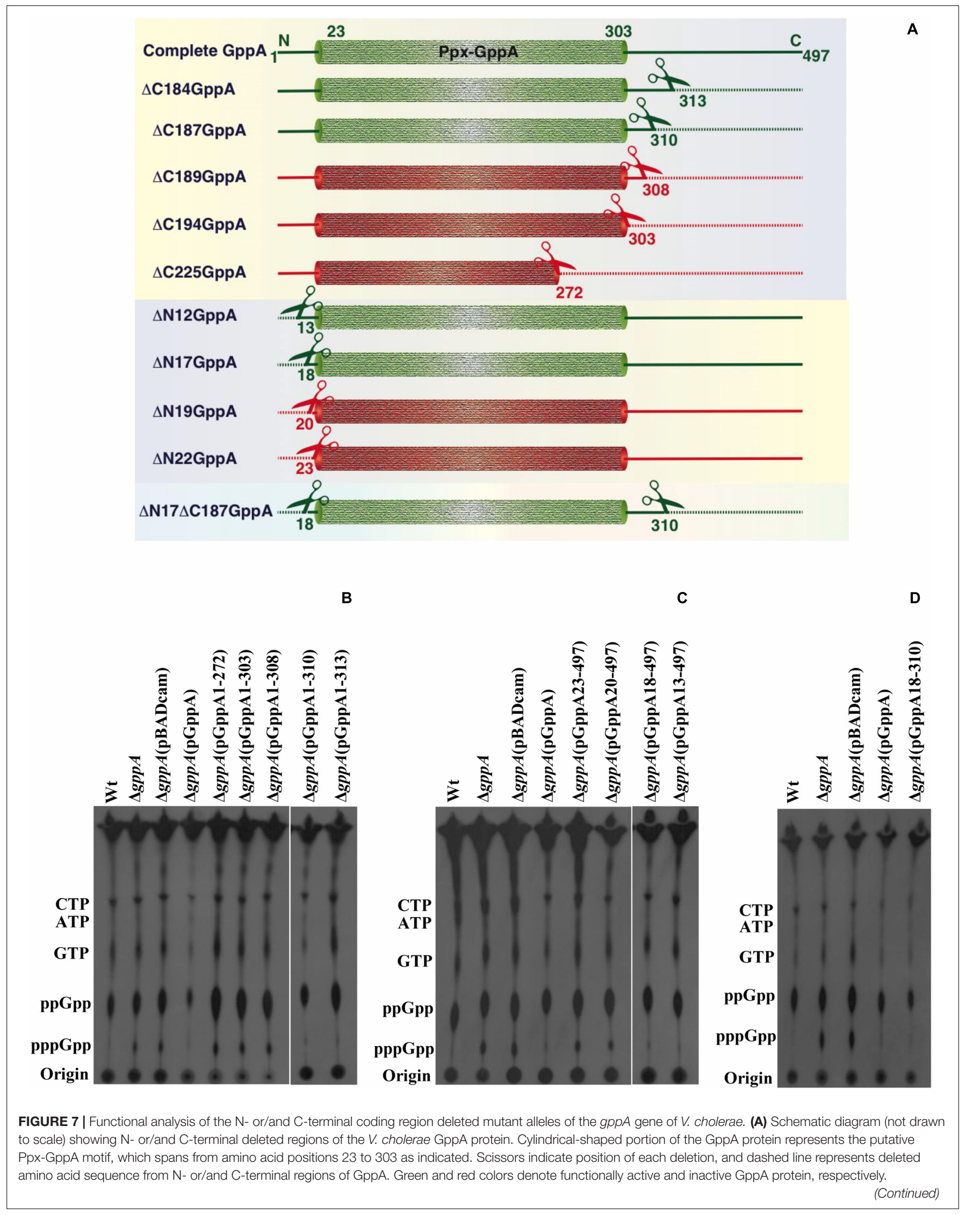




\section{FIGURE 7 | Continued}

$\mathrm{N}$ - and $\mathrm{C}$-terminally deleted amino acid sequence of the GppA protein is symbolized by $\Delta \mathrm{N}$ and $\Delta \mathrm{C}$, respectively, followed by the number of amino acids deleted as shown in the left margin. Intact GppA protein is 497 amino acids long, and the stretch of amino acid sequences present after deletion from $\mathrm{N}$ - or $\mathrm{C}$-terminal of the GppA protein are shown at the corresponding ends. (B) Complementation analysis using C-terminal encoding sequence deleted alleles. Each C-terminally truncated GppA protein was expressed using $0.2 \%$ arabinose as an inducer in the $\triangle g p p A$ mutant through the respective recombinant plasmid as shown above each lane. For the detection of pppGpp/ppGpp, thin layer chromatography (TLC) analysis of each strain was done followed by autoradiography. The presence of labeled nucleotides including pppGpp/ppGpp is indicated in the left margin. Autoradiogram shows complementation in $\triangle g p p A(p G p p A 1-310)$ and $\triangle g p p A(p G p p A 1-313)$ strains but not in $\triangle g p p A(p G p p A 1-272), \Delta g p p A(p G p p A 1-303)$, and $\triangle g p p A(p G p p A 1-308)$ strains. Wt, $\triangle g p p A, \Delta g p p A$ carrying the empty vector pBADcam $[\triangle g p p A(p B A D c a m)]$ and $\triangle g p p A$ carrying the full-length GppA expressing clone pGppA [ $\triangle$ gppA(pGppA)] were used as controls. (C) Complementation analysis using $\mathrm{N}$-terminal encoding sequence deleted alleles. Each $\mathrm{N}$-terminally truncated GppA protein was expressed using $0.2 \%$ arabinose as an inducer in the $\Delta$ gppA mutant through the respective recombinant plasmid as shown above each lane. TLC analysis of each strain was done followed by autoradiography. The presence of labeled nucleotides including pppGpp/ppGpp is indicated in the left margin. Autoradiogram shows complementation in strains $\Delta g p p A(p G p p A 18-497)$ and $\triangle g p p A(p G p p A 13-497)$ but not in strains $\triangle g p p A(p G p p A 23-497)$ and $\triangle g p p A(p G p p A 20-497)$. Control strains used as indicated in (B). (D) Determination of minimal functional region of the GppA protein. Here, both $\mathrm{N}$ - and $\mathrm{C}$-terminal coding regions deleted gppA clone pGppA18-310 was used for complementation in $\triangle$ gppA mutant. For the expression of the truncated GppA protein, 0.2\% arabinose was used. TLC analysis was done as described in (B). Strains used are Wt, $\triangle g p p A$, $\triangle g p p A(p B A D c a m), \triangle g p p A(p G p p A)$, and $\triangle g p p A(p G p p A 18-310)$. The presence of labeled nucleotides including pppGpp and ppGpp is indicated in the left margin.

\section{DISCUSSION}

The $V$. cholerae SR pathway gene $g p p A$, the product of which converts pppGpp to ppGpp (Figure 1), has not been characterized previously. Therefore, the main objective of this study was to characterize the $g p p A$ gene function by genetic and mutational approaches. We found that unlike in E. coli, the $g p p A$ gene in the genome of $V$. cholerae is arranged in an operon along with $r h l B$ (Figure 3). In E. coli, the $r h l B$ gene codes for an ATP-dependent RNA helicase, which is a component of the RNA degradasome complex (Khemici et al., 2005). Although at present no information is available about the function of RhlB of $V$. cholerae, the arrangement of the $r h l B-g p p A$ genes in an operon has raised a possibility that RhlB could be involved in the regulation of the SR in this pathogen, which needs investigation.

We found that the accumulation of pppGpp in $\triangle g p p A$ cells is high $(\sim 10$-fold) compared to the isogenic Wt strain during amino acid starvation but not under glucose deficient condition (Figures 4, 5). We have shown earlier that under amino acid starvation, $V$. cholerae RelA synthesizes (p)ppGpp (Haralalka et al., 2003), while during glucose starvation, SpoT and RelV enzymes are responsible for (p)ppGpp synthesis (Das and Bhadra, 2008; Das et al., 2009; Dasgupta et al., 2014). Therefore, it may be argued here that Wt cells produce both pppGpp and ppGpp using GTP and GDP as substrates, respectively, during amino acid starvation, but GppA most likely converts most of the pppGpp molecules to ppGpp (Figure 4). In contrast, under glucose starvation, there was no difference in $\mathrm{pppGpp/ppGpp}$ accumulation in Wt and $\triangle g p p A$ cells suggesting that the result is independent of GppA. It may be possible that the affinity of GppA to the pppGpp substrate is altered under glucose starvation stress, or may be some unknown factor is needed to activate the GppA enzyme under this condition, which needs further work. In this context, it may be noted that in E. coli, significant amounts of pppGpp accumulate in $\triangle g p p A$ mutant compared to an isogenic Wt strain under both amino acid and glucose starvation conditions (Somerville and Ahmed, 1979). However, our result on glucose starvation of $V$. cholerae cells does not support this finding in E. coli, which could be organism specific.

In this study, we have also characterized the $p p x$ gene of $V$. cholerae to determine whether it has any $g p p A$ like function. This is because both $\mathrm{Ppx}$ and GppA contain conserved Ppx-GppA motif, which appears to be needed for their similar enzymatic functions (Kristensen et al., 2008). Recently, it has been reported that deletion of the $p p x 1$ gene of the enteropathogen Campylobacter jejuni leads to increase in intracellular accumulation of pppGpp as well as polyP (Malde et al., 2014). We found that the overexpression of Ppx can complement the GppA function in amino acid starved $\triangle g p p A V$. cholerae cells (Figure 4). Similarly, Kuroda et al. (1997) have reported complementation of the GppA function in an E. coli $\triangle g p p A \Delta p p k x$ mutant strain (GppA-Ppk_Ppx-) by overexpressing the Ppx enzyme. Very recently, the structure of the Ppx/GppA protein of the Gram-negative bacterium Helicobacter pylori has been solved (Song et al., 2019). It was found that the H. pylori Ppx/GppA enzyme is $\sim 27$-fold less efficient in hydrolyzing pppGpp compared to its polyP hydrolase activity. Analysis of the crystal structures of Ppx and GppA also supports that the active site of Ppx can efficiently bind the narrower polyP but not the wider pppGpp molecule. Similarly, the structure of the active site of GppA allows pppGpp to bind efficiently and hydrolyze it to ppGpp (Song et al., 2019). Thus, it could be plausible that overexpression of $V$. cholerae Ppx in $\triangle g p p A$ cells may hydrolyze pppGpp and complement the $g p p A$ mutant phenotype due its probable weak pppGppase activity. However, further studies are needed to understand the exact role of Ppx in the complex metabolic network of (p)ppGpp in $V$. cholerae.

Currently, the expression profile of the $g p p A$ gene in different growth phases is not available for any Gramnegative bacteria. Such analysis indicated that in $V$. cholerae, the $g p p A$ gene expression is constitutive, although there is modest increase in transcripts level during the stationary growth phase (Figure 6A). This is likely since during stationary phase, bacterial cells face exhaustion of nutrients, which may trigger SR-related gene expression including $g p p A$. Since $g p p A$ expression during amino acid starvation has been observed to be high compared to that of glucose-starved cells (Figure 6B), which could be one of the reasons for no accumulation of pppGpp in amino-acid-starved Wt cells.

After establishing the function of the $g p p A$ gene, we determined the core functional region of the GppA protein of $V$. cholerae by progressive deletion analysis of the 
$\mathrm{N}$ - and C-terminal regions carrying the conserved domains (I-IV) and Ppx-GppA motif (Figure 2B). However, extensive complementation analysis of $\mathrm{N}$ - or/and C-terminal coding deletion alleles allowed us to validate that the Ppx-GppA motif of $V$. cholerae GppA actually spans from amino acid positions 18 to 310 residues, and this is the first report where we have determined the potential minimal functional boundaries of the GppA protein (Figure 7). It is important to mention here that the GppA protein of $A$. aeolicus, the crystal structure of which has been reported, is only 312 amino acids long. On the other hand, in Gram-negative bacteria including $V$. cholerae, GppA is composed of about 500 amino acids (Figure 2B). Our deletion analysis indicated that the Ppx-GppA motif containing the core functional region of $V$. cholerae GppA is 293 amino acids long, which is very close to that of $A$. aeolicus GppA complete protein. As mentioned, the presence of four distinct domains (I-IV) in E. coli Ppx and GppA have been reported (Rangarajan et al., 2006; Song et al., 2019). While domains I and II form the active sites of the Ppx and GppA proteins, domain III has similarity with the hydrolase domain of the SpoT enzyme and is probably responsible for the dimer formation of Ppx in E. coli (Rangarajan et al., 2006). On the other hand, domain IV showed structural similarities with the cold shock-associated RNA-binding protein (Rangarajan et al., 2006; Song et al., 2019). Our deletion analysis highlights particularly the importance of the conserved $\mathrm{N}$-terminal domains I and II of the GppA protein of $V$. cholerae because disruption of these domains but not the C-terminal domains III and IV leads to functional loss of GppA (Figure 7). Thus, it seems that similar functions are being carried out by the domains I and II of $V$. cholerae GppA protein.

In conclusion, the present study shows that the deletion of $V$. cholerae $g p p A$ gene leads to accumulation of substantial amounts of pppGpp molecules during amino-acid-starved condition. At present, however, it is not clearly known which of the alarmones, ppGpp or pppGpp, is most potent in regulating the different phenotypes of SR in bacteria including $V$. cholerae. Since ppGpp is synthesized directly from GDP and almost in all bacteria, the level of intracellular ppGpp under nutritional stress conditions is higher than pppGpp, it is likely that ppGpp is the key player of SR in bacteria (Cashel et al., 1996; Potrykus and Cashel, 2008; Sajish et al., 2009; Mechold et al., 2013). In addition, the presence of functional GppA in Gram-negative bacteria further supports the hypothesis that ppGpp is the critical molecule for controlling SR in bacteria. It also appears from

\section{REFERENCES}

Akiyama, M., Crooke, E., and Kornberg, A. (1993). An exopolyphosphatase of Escherichia coli. The enzyme and its ppx gene in a polyphosphate operon. J. Biol. Chem. 268, 633-639.

Ausubel, F. M., Brent, R., Kingston, R. E., Moore, D. D., Seidman, J. G., Smith, J. A., et al. (1989). Current Protocols in Molecular Biology. New York, NY: John Wiley and Sons.

Basu, P., and Bhadra, R. K. (2019). Post-transcriptional regulation of cholera toxin production in Vibrio cholerae by the stringent response regulator DksA. Microbiology 165, 102-112. doi: 10.1099/mic.0.000743 this study that the activation of the RelA and GppA enzymes are probably linked with amino acid starvation condition, which may help in maintaining the lower pppGpp level in $V$. cholerae. Further studies on other Gram-negative bacteria may help in establishing this unique link between RelA and GppA.

\section{DATA AVAILABILITY STATEMENT}

Data presented in this study are included in the article/Supplementary Material, further inquiries can be directed to the corresponding authors.

\section{AUTHOR CONTRIBUTIONS}

$\mathrm{RKB}$ and $\mathrm{BD}$ designed the study. $\mathrm{DR}$ and $\mathrm{SD}$ performed the experiments. $\mathrm{DR}, \mathrm{SD}, \mathrm{BD}$, and $\mathrm{RKB}$ analyzed the data. $\mathrm{DR}, \mathrm{BD}$, and RKB wrote the manuscript. All authors have contributed to the article and approved the submitted version.

\section{FUNDING}

The work was supported by the research grant (MLP118) of Council of Scientific and Industrial Research (CSIR) and also in part by Dept. of Biotechnology (DBT), Govt. of India (Grant No. BT/MB/THSTI/HMC-SFC/2011) to BD.

\section{ACKNOWLEDGMENTS}

We thank Pratap Koyal and Shibprasad Sharma for their excellent technical assistance in this work. DR is grateful for the research fellowship from the University Grant Commission (UGC), Government of India. SD is grateful for the research fellowship from CSIR.

\section{SUPPLEMENTARY MATERIAL}

The Supplementary Material for this article can be found online at: https://www.frontiersin.org/articles/10.3389/fmicb. 2020.564644/full\#supplementary-material

Basu, P., Pal, R. R., Dasgupta, S., and Bhadra, R. K. (2017). DksA-HapR-RpoS axis regulates haemagglutinin protease production in Vibrio cholerae. Microbiology 163, 900-910. doi: 10.1099/mic.0.000469

Cashel, M., Gentry, D. R., Hernandes, V. J., and Vinella, D. (1996). "The stringent respons," in Escherichia coli and Salmonella typhimurium: Cellular and Molecular Biology, 2nd Edn, Vol. 2, ed. F. C. Neidhardt (Washington, DC: ASM Press), 1458-1496.

Choi, M. Y., Wang, Y., Wong, L. L., Lu, B. T., Chen, W. Y., Huang, J. D., et al. (2012). The two PPX-GppA homologues from Mycobacterium tuberculosis have distinct biochemical activities. PLoS One 7:e42561. doi: 10.1371/journal.pone. 0042561 
Choy, H. E. (2000). The study of guanosine 5'-diphosphate 3'-diphosphatemediated transcription regulation in vitro using a coupled transcriptiontranslation system. J. Biol. Chem. 275, 6783-6789. doi: 10.1074/jbc.275.10. 6783

Dar, H. H., Prasad, D., Varshney, G. C., and Chakraborti, P. K. (2011). Secretory nucleoside diphosphate kinases from both intra- and extracellular pathogenic bacteria are functionally indistinguishable. Microbiology 15, 3024-3035. doi: 10.1099/mic.0.049221-0

Das, B., and Bhadra, R. K. (2008). Molecular characterization of Vibriocholerae $\Delta$ relA $\Delta$ spoT double mutants. Arch. Microbiol. 189, 227-238.

Das, B., Pal, R. R., Bag, S., and Bhadra, R. K. (2009). Stringent response in Vibrio cholerae: genetic analysis of spoT gene function and identification of a novel (p) ppGpp synthetase gene. Mol. Microbiol. 72, 380-398. doi: 10.1111/j.1365-2958. 2009.06653.x

Dasgupta, S., Basu, P., Pal, R. R., Bag, S., and Bhadra, R. K. (2014). Genetic and mutational characterization of the small alarmone synthetase gene relV of Vibrio cholerae. Microbiology 160, 1855-1866. doi: 10.1099/mic.0.079319-0

Gaca, A. O., Colomer-Winter, C., and Lemos, J. A. (2015). Many means to a common end: the intricacies of (p)ppGpp metabolism and its control of bacterial homeostasis. J. Bacteriol. 197, 1146-1156. doi: 10.1128/jb.02577-14

Hara, A., and Sy, J. (1983). Guanosine 5'-triphosphate, 3'-diphosphate 5'phosphohydrolase: purification and substrate specificity. J. Biol. Chem. 258, 1678-1683.

Haralalka, S., Nandi, S., and Bhadra, R. K. (2003). Mutation in the relA gene of Vibrio cholerae affects in vitro and in vivo expression of virulence factors. J. Bacteriol. 185, 4672-4682. doi: 10.1128/jb.185.16.4672-4682.2003

He, H., Cooper, J. N., Mishra, A., and Raskin, D. M. (2012). Stringent response regulation of biofilm formation in Vibrio cholerae. J Bacteriol. 194, 2962-2972. doi: $10.1128 /$ jb.00014- 12

Heidelberg, J. F., Eisen, J. A., Nelson, W. C., Clayton, R. A., Gwinn, M. L., Dodson, R. J., et al. (2000). DNA sequence of both chromosomes of the cholera pathogen Vibrio cholerae. Nature 406, 477-483.

Keasling, J. D., Bertsch, L., and Kornberg, A. (1993). Guanosine pentaphosphate phosphohydrolase of Escherichia coli is a long-chain exopolyphosphatase. Proc. Natl. Acad. Sci. U.S.A. 90, 7029-7033. doi: 10.1073/pnas.90.15.7029

Khemici, V., Poljak, L., Toesca, I., and Carpousis, A. J. (2005). Evidence in vivo that the DEAD-box RNA helicase RhlB facilitates the degradation of ribosomefree mRNA by RNaseE. Proc. Natl. Acad. Sci. U.S.A. 102, 6913-6918. doi: 10.1073/pnas.0501129102

Koonin, E. V. (1994). Yeast protein controlling inter-organelle communication is related to bacterial phosphatases containing the Hsp 70-type ATP-binding domain. Trends Biochem Sci. 19, 156-157. doi: 10.1016/0968-0004(94)90275-5

Kristensen, O., Laurberg, M., Liljas, A., Kastrup, J. S., and Gajhede, M. (2004). Structural characterization of the stringent response related exopolyphosphatase/guanosine pentaphosphate phosphohydrolase protein family. Biochemistry 43, 8894-8900. doi: 10.1021/bi049083c

Kristensen, O., Ross, B., and Gajhede, M. (2008). Structure of the PPX/GPPA phosphatase from Aquifex aeolicus in complex with the alarmone ppGpp. J. Mol. Biol. 375, 1469-1476. doi: 10.1016/j.jmb.2007.11.073

Kudrin, P., Dzhygyr, I., Ishiguro, K., Beljantseva, J., Maksimova, E., Oliveira, S. R. A., et al. (2018). The ribosomal A-site finger is crucial for binding and activation of the stringent factor RelA. Nucleic Acids Res. 46, 1973-1983. doi: 10.1093/nar/gky023

Kuroda, A., Murphy, H., Cashel, M., and Kornberg, A. (1997). Guanosine tetraand pentaphosphate promote accumulation of inorganic polyphosphate in Escherichia coli. J. Biol. Chem. 272, 21240-21243. doi: 10.1074/jbc.272.34.21240

Kuroda, A., Nomura, K., Ohtomo, R., Kato, J., Ikeda, T., Takiguchi, N., et al. (2001). Role of inorganic polyphosphate in promoting ribosomal protein degradation by the Lon protease in E. coli. Science 293, 705-708. doi: $10.1126 /$ science. 1061315

Malde, A., Gangaiah, D., Chandrashekhar, K., Pina-Mimbela, R., Torrelles, J. B., and Rajashekara, G. (2014). Functional characterization of exopolyphosphatase/guanosine pentaphosphate phosphohydrolase (PPX/GPPA) of Campylobacter jejuni. Virulence 5, 521-533. doi: $10.4161 /$ viru. 28311
Mechold, U., Murphy, H., Brown, L., and Cashel, M. (2002). Intramolecular regulation of the opposing (p)ppGpp catalytic activities of Rel(Seq), the Rel/Spo enzyme from Streptococcus equisimilis. J. Bacteriol. 184, 2878-2888. doi: 10. 1128/jb.184.11.2878-2888.2002

Mechold, U., Potrykus, K., Murphy, H., Murakami, K. S., and Cashel, M. (2013). Differential regulation by ppGpp versus pppGpp in Escherichia coli. Nucleic Acids Res. 41, 6175-6189. doi: 10.1093/nar/gkt302

Ogawa, N., Tzeng, C. M., Fraley, C. D., and Kornberg, A. (2000). Inorganic polyphosphate in Vibrio cholerae: genetic, biochemical, and physiologic features. J. Bacteriol. 182, 6687-6693. doi: 10.1128/jb.182.23.6687-6693.2000

Pal, R. R., Bag, S., Dasgupta, S., Das, B., and Bhadra, R. K. (2012). Functional characterization of the stringent response regulatory gene $d k s A$ of Vibrio cholerae and its role in modulation of virulence phenotypes. J. Bacteriol. 194, 5638-5648. doi: 10.1128/jb.00518-12

Pal, R. R., Das, B., Dasgupta, S., and Bhadra, R. K. (2011). Genetic components of stringent response in Vibrio cholerae. Indian J. Med. Res. 133, 212-217.

Potrykus, K., and Cashel, M. (2008). (p)ppGpp: still magical? Annu. Rev. Microbiol. 62, 35-51. doi: 10.1146/annurev.micro.62.081307.162903

Rangarajan, E. S., Nadeau, G., Li, Y., Wagner, J., Hung, M. N., Schrag, J. D., et al. (2006). The structure of the exopolyphosphatase (PPX) from Escherichia coli O157:H7 suggests a binding mode for long polyphosphate chains. J. Mol. Biol. 359, 1249-1260. doi: 10.1016/j.jmb.2006.04.031

Reizer, J., Reizer, A., Saier, M. H. Jr., Bork, P., and Sander, C. (1993). Exopolyphosphate phosphatase and guanosine pentaphosphate phosphatase belong to the sugar kinase/actin/hsp 70 superfamily. Trends Biochem. Sci. 18, 247-248. doi: 10.1016/0968-0004(93)90172-j

Sajish, M., Kalayil, S., Verma, S. K., Nandicoori, V. K., and Prakash, B. (2009). The significance of EXDD and RXKD motif conservation in Rel proteins. J. Biol. Chem. 284, 9115-9123. doi: 10.1074/jbc.m807187200

Seyfzadeh, M., Keener, J., and Nomura, M. (1993). spoT-dependent accumulation of guanosine tetraphosphate in response to fatty acid starvation in Escherichia coli. Proc. Natl. Acad. Sci. U.S.A. 90, 11004-11008. doi: 10.1073/pnas.90.23. 11004

Somerville, C. R., and Ahmed, A. (1979). Mutants of Escherichia coli defective in the degradation of guanosine 5'-triphosphate, 3'-diphosphate (pppGpp). Mol. Gen. Genet. 169, 315-323. doi: 10.1007/bf00382277

Song, H., Dharmasena, M. N., Wang, C., Shaw, G. X., Cherry, S., Tropea, J. E., et al. (2019). Structure and activity of PPX/GppA homologs from Escherichia coli and Helicobacter pylori. FEBS J. 287, 1865-1885. doi: 10.1111/febs.15120

Stephens, J. C., Artz, S. W., and Ames, B. N. (1975). Guanosine 5'-diphosphate 3'-diphosphate (ppGpp): positive effector for histidine operon transcription and general signal for amino-acid deficiency. Proc. Natl. Acad. Sci. U.S.A. 72, 4389-4393. doi: 10.1073/pnas.72.11.4389

Verma, J., Bag, S., Saha, B., Kumar, P., Ghosh, T. S., Dayal, M., et al. (2019). Genomic plasticity associated with antimicrobial resistance in Vibrio cholerae. Proc. Natl. Acad. Sci. U.S.A. 116, 6226-6231.

Wang, J. D., Sanders, G. M., and Grossman, A. D. (2007). Nutritional control of elongation of DNA replication by (p)ppGpp. Cell 128, 865-875. doi: 10.1016/j. cell.2006.12.043

Xiao, H., Kalman, M., Ikehara, K., Zemel, S., Glaser, G., and Cashel, M. (1991). Residual guanosine 3', 5'-bispyrophosphate synthetic activity of relA null mutants can be eliminated by spoT null mutations. J. Biol. Chem. 266, 59805990.

Conflict of Interest: The authors declare that the research was conducted in the absence of any commercial or financial relationships that could be construed as a potential conflict of interest.

Copyright (c) 2020 Rakshit, Dasgupta, Das and Bhadra. This is an open-access article distributed under the terms of the Creative Commons Attribution License (CC BY). The use, distribution or reproduction in other forums is permitted, provided the original author(s) and the copyright owner(s) are credited and that the original publication in this journal is cited, in accordance with accepted academic practice. No use, distribution or reproduction is permitted which does not comply with these terms. 